\title{
Two Techniques for Compensating the Probe Positioning Errors in the Spherical NF-FF Transformation for Elongated Antennas
}

\author{
F. D’Agostino, F. Ferrara, C. Gennarelli', R. Guerriero and M. Migliozzi \\ D.I.E.I.I - University of Salerno, via Ponte Don Melillo, 84084 Fisciano (Salerno), Italy
}

\begin{abstract}
Two effective approaches for compensating the positioning errors in a near-field-far-field (NF-FF) transformation technique with spherical scanning for long antennas using a nonredundant number of data are presented. The transformation technique relies on the nonredundant sampling representations of the electromagnetic fields and on the optimal sampling interpolation (OSI) expansions, and assumes the antenna under test as enclosed in a prolate ellipsoid, a source modelling particularly suitable to deal with elongated antennas. In order to evaluate the NF data at the points fixed by the nonredundant representation from the acquired irregularly spaced ones, the former approach employs the singular value decomposition method, whereas the latter makes use of an iterative technique. The former can be applied when the irregularly samples lie on nonuniform parallels, thus allowing to reduce the starting two-dimensional problem into two independent one-dimensional ones. The latter can be employed also when such a hypothesis does not hold, but requires the existence of a one-to-one correspondence associating at each uniform sampling point the nearest irregular one. In both the cases, the NF data needed by a probe compensated NF-FF transformation with spherical scanning are efficiently evaluated by using an OSI algorithm. Numerical tests assessing the effectiveness of the proposed approaches and their stability with respect to random errors affecting the NF data are shown.
\end{abstract}

Keywords: NF-FF transformations, spherical scanning, nonredundant sampling representations of electromagnetic fields, probe positioning error compensation.

\section{INTRODUCTION}

Among the near-field-far-field (NF-FF) transformation techniques, that using the spherical scanning is particularly attractive, since it allows the reconstruction of the full radiation pattern of the antenna under test (AUT) from a single set of NF measurements [1-10]. However, the data processing is considerably more complex than that required by planar and cylindrical NF facilities. In [8], the classical NF-FF transformation with spherical scanning [1] has been modified by taking into account the spatial bandlimitation properties of the electromagnetic (EM) fields [11]. In particular, the choice of the highest spherical wave to be considered has been rigorously fixed by the bandlimitation properties and no longer determined according to a rule-of-thumb related to the smallest sphere containing the AUT. Moreover, the number of data on the parallels has resulted to be decreasing towards the poles. In the same paper, the nonredundant sampling representations of the EM field [12] have been applied to reduce in a significant way the number of needed NF data when dealing with an antenna having one or two predominant dimensions, which has been considered as enclosed in a prolate or oblate ellipsoid, respectively. Then, an optimal sampling interpolation (OSI) formula, which allows the reconstruction of the data required by the aforementioned NFFF transformation from a nonredundant number of $\mathrm{NF}$

*Address correspondence to this author at the Dipartimento di Ingegneria Elettronica e Ingegneria Informatica University of Salerno Via Ponte Don Melillo 84084 Fisciano (SA) Italy; Tel: +39 089-964297;

Fax: +39 089-964218; E-mail: gennar@diiie.unisa.it samples, has been developed. In [9], the ideal probe assumption made in [8] has been removed by proposing an efficient probe compensated NF-FF transformation with spherical scanning tailored for elongated or quasi-planar antennas. At last, effective NF-FF transformations with spherical scanning tailored for nonspherical antennas and based on very flexible AUT modellings have been developed in [10]. In particular, a cylinder ended in two half-spheres has been adopted to shape an electrically long antenna, whereas a quasi-planar antenna has been considered as enclosed in a surface formed by two circular bowls with the same aperture diameter but different lateral bends.

Unfortunately, the errors due to an inaccurate control of the positioning systems prevent the possibility to get regularly spaced NF measurements, even though their position can be accurately read by optical devices. In addition, the finite resolution of the positioning devices and their imprecise synchronization do not allow one to exactly locate the probe at the points fixed by the sampling representation. Therefore, the development of an effective reconstruction algorithm from irregularly spaced data becomes relevant. A procedure based on the conjugate gradient iteration method and employing the unequally spaced fast Fourier transform $[13,14]$ has been proposed in the standard planar [15] and spherical [16] scannings. However, such a procedure is not suitable for scanning techniques taking advantage of the nonredundant sampling representations of EM fields, wherein the "a priori" information on the AUT and proper OSI formulas are exploited to reconstruct the NF data required by the corresponding classical NF-FF transformation. As it has 


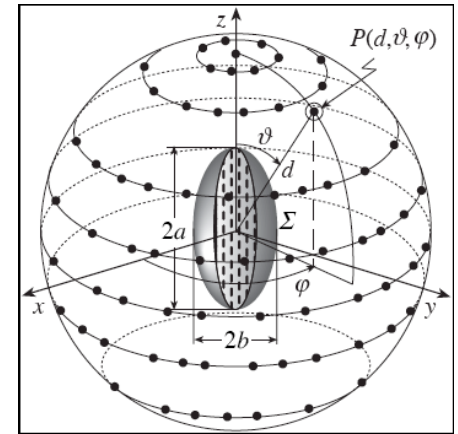

Fig. (1). Geometry of the problem.

been clearly stressed in [17], wherein a more comprehensive discussion can be found, the formulas available in literature for the direct reconstruction from nonuniform samples are valid only for particular sampling points distributions, are not user friendly and unstable. A feasible and convenient strategy is to recover the uniform samples from those nonuniformly spaced and then determine the value at any point of the scanning surface by an accurate and stable OSI formula. Two approaches [17-20] have been proposed to this end and compared and experimentally validated in the cylindrical scanning case [21]. The former [17, 18] is based on an iterative technique which converges only if it is possible to build a biunique correspondence associating at each uniform sampling point the nearest nonuniform one. The latter [19, 20] makes use of the singular value decomposition (SVD) method [22] and can be conveniently applied when the starting two-dimensional problem can be reduced to find the solution of two independent one-dimensional ones. If this is not the case, the dimension of the involved matrixes would become very large, thus requiring a massive computational effort. This occurs, f.i., in the helicoidal scanning, where, in presence of positioning errors, the acquired NF data are no longer on a helix. Accordingly, in such a case, the iterative technique can be conveniently employed [23].

The aim of this paper is just the application of the abovementioned approaches to the compensation of the probe positioning errors in the NF-FF transformation with spherical scanning $[8,9]$. For space saving, only the prolate ellipsoidal modelling of the AUT will be considered in the following, so that the resulting NF-FF transformation from nonuniformly distributed spherical NF data is tailored for electrically long antennas. The extension to the case of the oblate modelling of the AUT being straightforward.

\section{NONREDUNDANT SAMPLING REPRESENTA- TION OF THE VOLTAGE}

Since, as shown in [24], the voltage $V$ measured by a nondirective probe has the same effective spatial bandwidth of the AUT field, the nonredundant sampling representations of EM fields [12] can be applied to such a voltage. Let us consider an electrically long AUT, a probe scanning a spherical surface of radius $d$ in the NF region, and adopt the spherical coordinate system $(r, \vartheta, \varphi)$ to denote an observation point. An effective source modelling for such a kind of antenna is obtained by choosing the surface $\Sigma$ (enclosing it) coincident with the smallest prolate ellipsoid having major and minor semi-axes equal to $a$ and $b$ (Fig. 1).
According to [12], let us introduce the probe "reduced voltage"

$\tilde{V}(\xi)=V(\xi) \mathrm{e}^{\mathrm{j} \gamma(\xi)}$

where $\gamma(\xi)$ is a phase function to be determined and $\xi$ is an optimal parameter used to describe each of the curves $C$ (meridians and parallels) representing the spherical surface. The error occurring when $\tilde{V}$ is approximated by a bandlimited function becomes negligible as the bandwidth exceeds a critical value $W_{\xi}[12]$, so that it can be effectively controlled by choosing a bandwidth equal to $\chi^{\prime} W_{\xi}, \chi^{\prime}>1$ being an excess bandwidth factor. When $C$ is a meridian, by adopting $W_{\xi}=\beta \ell^{\prime} / 2 \pi \quad(\beta \quad$ is the wavenumber and $\ell^{\prime}=4 a E\left(\pi / 2 \mid \varepsilon^{2}\right)$ is the length of the ellipse $C^{\prime}$, intersection curve between the meridian plane through the observation point $P$ and $\Sigma$ ), we get $[8,12]$ :

$$
\begin{aligned}
& \gamma=\beta a\left[v \sqrt{\frac{v^{2}-1}{v^{2}-\varepsilon^{2}}}-E\left(\cos ^{-1} \sqrt{\frac{1-\varepsilon^{2}}{v^{2}-\varepsilon^{2}}} \mid \varepsilon^{2}\right)\right] \\
& \xi=\frac{\pi}{2}\left[1+\frac{E\left(\sin ^{-1} u \mid \varepsilon^{2}\right)}{E\left(\pi / 2 \mid \varepsilon^{2}\right)}\right]
\end{aligned}
$$

where $E(\cdot \mid \cdot)$ denotes the elliptic integral of second kind, $\varepsilon=f / a$ is the eccentricity of $C^{\prime}, 2 f$ is its focal distance and $u=\left(r_{1}-r_{2}\right) / 2 f$ and $v=\left(r_{1}+r_{2}\right) / 2 a$ are the elliptic coordinates, $r_{1,2}$ being the distances from the observation point $P$ to the foci of $C^{\prime}$. Relation (3) is valid for $\vartheta$ belonging to the range $[0, \pi / 2]$. The case $\vartheta$ belonging to $[\pi / 2, \pi]$ can be handled by determining the value $\xi^{\prime}$ corresponding to the point specified by the angle $\pi-\vartheta$ and then putting $\xi=\pi-\xi^{\prime}$. As shown in [12], the curves $\gamma=$ const and $\xi=$ const are ellipses and hyperbolas confocal to $C^{\prime}$. When the curve $C$ is a parallel at $\vartheta(\xi)$, the phase function is constant, the optimal parameter is the azimuthal angle $\varphi$ and the corresponding bandwidth is, $W_{\varphi}(\xi)=\beta b \sin \vartheta_{\infty}(\xi)$ $\vartheta_{\infty}=\sin ^{-1}(u)+\pi / 2$ being the polar angle of the asymptote to the hyperbola through $P[8,12]$.

According to the above results, the reduced voltage at $P$ on the meridian fixed by $\varphi$ can be evaluated via the following OSI expansion

$\tilde{V}(\xi(\vartheta), \varphi)=\sum_{n=n_{0}-q+1}^{n_{0}+q} \tilde{V}\left(\xi_{n}, \varphi\right) \Omega_{N}\left(\xi-\xi_{n}\right) D_{N "}\left(\xi-\xi_{n}\right)$

where $n_{0}=n_{0}(\xi)=\operatorname{Int}(\xi / \Delta \xi)$ is the index of the sample nearest to the output point, $2 q$ is the number of retained intermediate samples $\tilde{V}\left(\xi_{n}, \varphi\right)$, i.e., the reduced voltages at the intersection points between the sampling parallels and the considered meridian, and 


$$
\begin{aligned}
D_{N^{\prime \prime}}(\xi) & =\frac{\sin \left[\left(2 N^{\prime \prime}+1\right) \xi / 2\right]}{\left(2 N^{\prime \prime}+1\right) \sin (\xi / 2)} \\
\Omega_{N}(\xi) & =\frac{T_{N}\left[2 \cos ^{2}(\xi / 2) / \cos ^{2}\left(\xi_{0} / 2\right)-1\right]}{T_{N}\left[2 / \cos ^{2}\left(\xi_{0} / 2\right)-1\right]}
\end{aligned}
$$

are the Dirichlet and Tschebyscheff Sampling functions. Moreover, $\operatorname{Int}(x)$ denotes the integer part of $x$,

$$
\begin{aligned}
& \xi_{n}=n \Delta \xi=2 \pi n /\left(2 N^{\prime \prime}+1\right) ; N^{\prime \prime}=\operatorname{Int}\left(\chi N^{\prime}\right)+1 ; \\
& N^{\prime}=\operatorname{Int}\left(\chi^{\prime} W_{\xi}\right)+1 ; N=N^{\prime \prime}-N^{\prime}
\end{aligned}
$$

$\chi$ being the oversampling factor needed to control the truncation error. In (6), $T_{N}(\cdot)$ is the Tschebyscheff polynomial of degree $N$ and $\xi_{0}=q \Delta \xi$.

The intermediate samples are given by:

$$
\tilde{V}\left(\xi_{n}, \varphi\right)=\sum_{m=m_{0}-p+1}^{m_{0}+p} \tilde{V}\left(\xi_{n}, \varphi_{m, n}\right) \cdot \Omega_{M_{n}}\left(\varphi-\varphi_{m, n}\right) D_{M_{n}^{\prime \prime}}\left(\varphi-\varphi_{m, n}\right)
$$

where $\tilde{V}\left(\xi_{n}, \varphi_{m, n}\right)$ are the reduced voltage samples, uniformly spaced on the parallel at $\vartheta\left(\xi_{n}\right), 2 p$ is the retained samples number, $m_{0}=m_{0}(\varphi)=\operatorname{Int}\left(\varphi / \Delta \varphi_{n}\right)$, and

$$
\begin{aligned}
& \varphi_{m, n}=m \Delta \varphi_{n}=2 m \pi /\left(2 M_{n}^{\prime \prime}+1\right) \\
& M_{n}^{\prime \prime}=\operatorname{Int}\left(\chi M_{n}^{\prime}\right)+1 ; M_{n}^{\prime}=\operatorname{Int}\left(\chi^{*} W_{\varphi}\left(\xi_{n}\right)\right)+1 \\
& \chi^{*}=1+\left(\chi^{\prime}-1\right)\left[\sin \vartheta_{\infty}\left(\xi_{n}\right)\right]^{-2 / 3} ; M_{n}=M_{n}^{\prime \prime}-M_{n}^{\prime} ;
\end{aligned}
$$

By using the OSI expansions (4) and (9), it is possible to evaluate the probe voltage at any point on spherical surface and, in particular, at the points required by the classical NFFF transformation with spherical scanning [1] as modified in [8-10].

\section{RECONSTRUCTION OF THE UNIFORM SAMPLES}

Two different approaches for reconstructing the uniformly spaced samples from the irregularly distributed ones will be described in this section by highlighting all their features.

\subsection{The SVD Approach}

Let us now assume that, apart from the sample at the pole $\vartheta=0$, the irregularly distributed samples lie on parallels not regularly spaced (see Fig. 1). This hypothesis can really represent the spatial distribution of the NF measurements when the acquisition is made by parallels, as needed to exploit the reduction in the number of NF data on noncentral parallels, made possible by the nonredundant sampling representation. Accordingly, the considered two-dimensional problem can be split in two independent one-dimensional ones. In this framework, let us assume to know the probe voltage at $J_{k} \geq 2 M_{k}^{\prime \prime}+1$ nonuniform sampling points $\left(\eta_{k}, \phi_{j}\right)$ on the nonuniform parallel at $\vartheta\left(\eta_{k}\right), 2 M_{k}^{\prime \prime}+1$ being the number of the corresponding uniform sampling points $\varphi_{m, k}=m \Delta \varphi_{k}=2 m \pi /\left(2 M_{k}^{\prime \prime}+1\right)$. The reduced voltage $\tilde{V}$ at each nonuniform sampling point can be expressed via the OSI expansion (9), thus obtaining the linear system:

$$
\tilde{V}\left(\eta_{k}, \phi_{j}\right)=\sum_{m=m_{0}-p+1}^{m_{0}+p} \tilde{V}\left(\eta_{k}, \varphi_{m, k}\right) \Omega_{M_{k}\left(\phi_{j}-\varphi_{m, k}\right)} D_{M_{k}^{\prime \prime}\left(\phi_{j}-\varphi_{m, k}\right) ; j=1, \ldots, J_{k}}
$$

The overdetermined linear system (13) can be expressed in matrix form as $\underline{\underline{A}} \underline{x}=\underline{b}$, where $\underline{b}$ is the sequence $\tilde{V}\left(\eta_{k}, \phi_{j}\right)$ of the known nonuniform samples, $\underline{x}$ is the sequence of the unknown uniformly distributed ones $\tilde{V}\left(\eta_{k}, \varphi_{m, k}\right)$, and $\underline{\underline{A}}$ is the $J_{k} \times\left(2 M_{k}^{\prime \prime}+1\right)$ matrix, whose elements are given by the weight functions in the considered OSI expansion:

$a_{j m}=\Omega_{M_{k}}\left(\phi_{j}-\varphi_{m, k}\right) D_{M_{k}^{\prime \prime}}\left(\phi_{j}-\varphi_{m, k}\right)$

It is useful to note that, for a fixed row $j$, the elements of the matrix are equal to zero if the index $m$ is out of the range $\left[m_{0}\left(\phi_{j}\right)-p+1, m_{0}\left(\phi_{j}\right)+p\right]$. A solution, which is the best approximation in the least squares sense of the linear system (13), is obtained by using the SVD method [22].

Let us now tackle the problem of evaluating the probe voltage at a point $P(\vartheta, \varphi)$ on the sphere from the knowledge of the recovered uniform samples on the irregularly spaced parallels. To this end, the OSI expansion (9) can be employed to determine the intermediate samples $\tilde{V}\left(\eta_{k}, \varphi\right)$ on the meridian through $P$. Since these intermediate samples are nonuniformly distributed on the considered meridian, the voltage at $P$ can be found in analogous way by recovering the regularly spaced intermediate samples again via SVD and then interpolating them by means of (4).

It must be stressed that both the displacements between the uniform and nonuniform samples on the nonuniform parallels and those between the uniform and nonuniform parallels are assumed such that to each uniform sampling position must correspond at least a nonuniform one whose distance is less than one half the uniform sampling spacing $\left(\Delta \varphi_{k}\right.$ or $\Delta \xi$ ) to avoid a strong ill-conditioning of the related linear system $[19,20]$. Moreover, in order to minimize the computational effort for reconstructing the uniformly spaced samples on the uniform parallels, it is convenient to determine the same number $N_{S}$ of samples on each of them. This number is fixed according to the sampling rate on the equator. In such a way, although the so recovered NF data are slightly redundant in $\varphi$, the number of SVD relevant to the meridians is minimized being these samples aligned. It is worthy to note that the overall number of SVD required to recover them is $N_{S}$ plus the number of nonuniform parallels. Once these samples have been determined, the data required by the NF-FF transformation with spherical scanning can be evalu- 


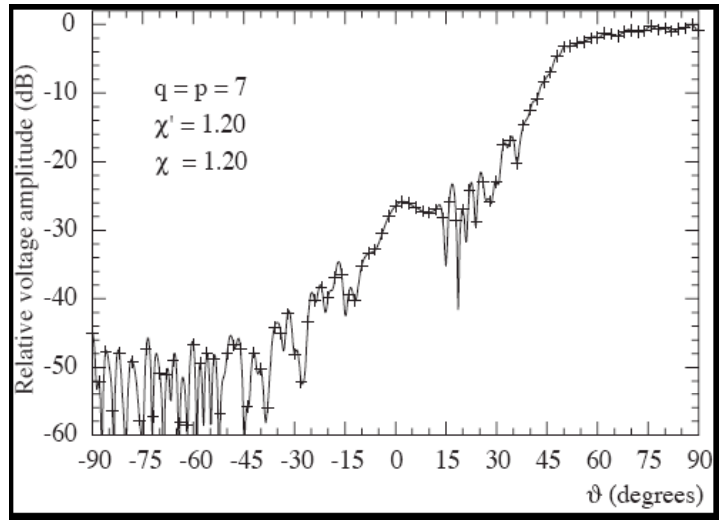

Fig. (2). Amplitude of the probe voltage $V^{\prime}$ on the meridian at $\varphi=90^{\circ}$. Solid line: exact. Crosses: reconstructed from nonuniform samples via the SVD based algorithm.

ated by using the OSI expansions (4) and (9), this last properly modified to take into account the redundancy in $\varphi$.

When removing the hypothesis that the nonuniformly distributed samples lie on parallels not regularly spaced, the two-dimensional problem can no longer be reduced to find the solution of two independent one-dimensional problems. In such a case, the SVD-based approach could be still used, but the dimension of the involved matrix would become very large, thus requiring a huge computational effort. Accordingly, it is convenient to resort to the iterative technique [17, 18 ].

\subsection{The Iterative Approach}

Let us assume in the following that the nonuniformly distributed samples are such that it is possible to build a one-toone correspondence associating at each uniform sampling point the "nearest" nonuniform one. In such a case, by expressing the reduced voltage at each nonuniform sampling point $\left(\eta_{k}, \phi_{j, k}\right)$ as a function of the unknown values at the nearest uniform ones $\left(\xi_{n}, \varphi_{m, n}\right)$ by using the OSI expansions (4) and (9), it results:

$$
\begin{gathered}
\tilde{V}\left(\eta_{k}, \phi_{j, k}\right)=\sum_{n=n_{0}-q+1}^{n_{0}+q}\left\{\Omega_{N}\left(\eta_{k}-\xi_{n}\right) D_{N^{\prime \prime}}\left(\eta_{k}-\xi_{n}\right) \cdot\right. \\
\quad \sum_{m=m_{0}-p+1}^{m_{0}+p} \tilde{V}\left(\xi_{n}, \varphi_{m, n}\right) \Omega_{M_{n}}\left(\phi_{j, k}-\varphi_{m, n}\right) \cdot \\
\left.\cdot D_{M_{n}^{\prime \prime}}\left(\phi_{j, k}-\varphi_{m, n}\right)\right\}
\end{gathered}
$$

The resulting linear system can be again rewritten in the matrix form $\underline{\underline{A}} \underline{x}=\underline{b}$, where now $\underline{b}$ is the sequence $\tilde{V}\left(\eta_{k}, \phi_{j, k}\right)$ of the known nonuniform samples, $\underline{x}$ is the sequence of the unknown uniformly distributed ones $\tilde{V}\left(\xi_{n}, \varphi_{m, n}\right)$, and $\underline{\underline{A}}$ is a $Q \times Q$ sparse matrix whose elements are given by the weight functions in the considered
OSI expansion, $Q$ being the overall number of the nonuniform/uniform samples. By splitting the matrix $\underline{\underline{A}}$ into its diagonal part $\underline{\underline{A}}_{D}$ and nondiagonal one $\underline{\underline{\Delta}}$, multiplying both members of the relation $\underline{\underline{A}} \underline{x}=\underline{b}$ by $\underline{\underline{A}}_{D}^{-1}$ and rearranging the terms, the following iterative procedure results:

$$
\underline{x}^{(v)}=\underline{\underline{A}}_{D}^{-1} \underline{b}-\underline{\underline{A}}_{D}^{-1} \underline{\underline{\Delta}} \underline{x}^{(v-1)}=\underline{x}^{(0)}-\underline{\underline{A}}_{D}^{-1} \underline{\underline{\Delta}} \underline{x}^{(v-1)}
$$

where $\underline{x}^{(v)}$ is the vector of the uniform samples estimated at the $v$ th step. Necessary conditions for the convergence of such an iterative algorithm are that the modulus of each element on the principal diagonal of the matrix $\underline{A}$ be not zero and greater than those of the other elements on the same row and column. These conditions are certainly fulfilled in the considered hypothesis of biunique correspondence between each uniform sampling point and the "nearest" nonuniform one. By straightforward evaluations, we finally get:

$$
\begin{aligned}
& \tilde{V}^{(v)}\left(\xi_{n}, \varphi_{m, n}\right)=\frac{1}{\Omega_{N}\left(\eta_{n}-\xi_{n}\right) D_{N^{\prime \prime}}\left(\eta_{n}-\xi_{n}\right)} . \\
& \cdot \frac{1}{\Omega_{M_{n}}\left(\phi_{m, n}-\varphi_{m, n}\right) D_{M_{n}^{\prime \prime}}\left(\phi_{m, n}-\varphi_{m, n}\right)}\left\{\tilde{V}\left(\eta_{n}, \phi_{m, n}\right)+\right. \\
& -\sum_{\ell=\ell_{0}-q+1}^{\ell_{0}+q} \sum_{i=i_{0}-p+1}^{i_{0}+p} \Omega_{N}\left(\eta_{n}-\xi_{\ell}\right) D_{N^{\prime \prime}}\left(\eta_{n}-\xi_{\ell}\right) \\
& \cdot \Omega_{M_{\ell}\left(\phi_{m, n}-\varphi_{i, \ell}\right) D_{M_{\ell}}\left(\phi_{m, n}-\varphi_{i, \ell}\right) \tilde{V}^{(v-1)}\left(\xi_{\ell}, \varphi_{i, \ell}\right)}
\end{aligned}
$$

Once the uniform samples have been so retrieved, the OSI expansions (4) and (9) can be used to reconstruct the NF data needed by the classical NF-FF transformation with spherical scanning [1] as modified in [8-10].

\section{NUMERICAL SIMULATIONS}

The effectiveness and robustness of the proposed algorithms to compensate the probe positioning errors in the NFFF transformation with spherical scanning have been assessed by many numerical tests. The reported simulations refer to a uniform planar array of $\lambda / 2$ spaced elementary Huygens sources polarized along the $z$ axis ( $\lambda$ being the wavelength). This array covers an elliptical zone in the plane $y=0$, with major and minor semi-axes equal to $20 \lambda$ and $5 \lambda$, respectively, so that such a long antenna can be very well fitted by a prolate ellipsoid. An open-ended circular waveguide with radius $0.338 \lambda$ is considered as probe and a sphere of radius $d=25 \lambda$ as scanning surface.

In the first set of figures (from Fig. 2 to Fig. 9), the nonuniformly distributed sampling points lie on parallels not regularly spaced. In particular, the NF data have been generated in such a way that the distance between the position of each nonuniform parallel and the associated uniform one is a random variable uniformly distributed in $(-\Delta \xi / 2, \Delta \xi / 2)$. In a similar way, the distances between the nonuniform sampling points and the corresponding uniform ones on each of 


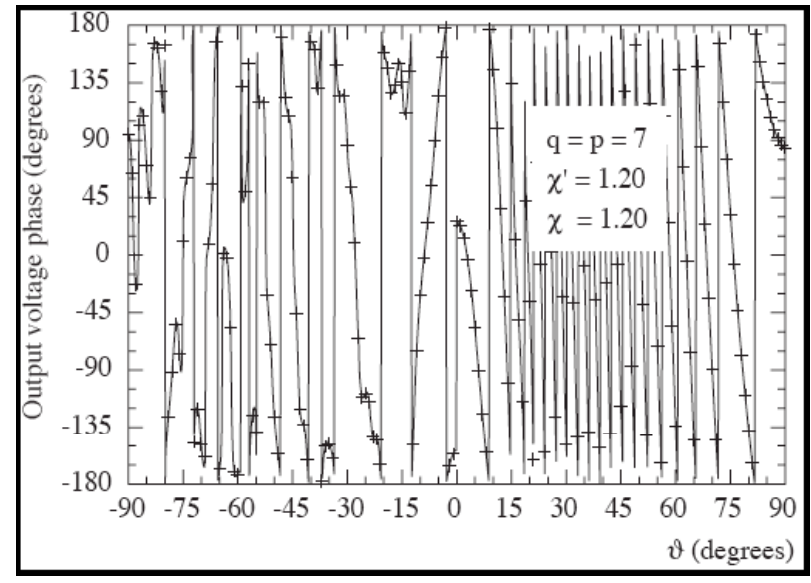

Fig. (3). Phase of the probe voltage $V^{\prime}$ on the meridian at $\varphi=90^{\circ}$. Solid line: exact. Crosses: reconstructed from nonuniform samples via the SVD based algorithm.

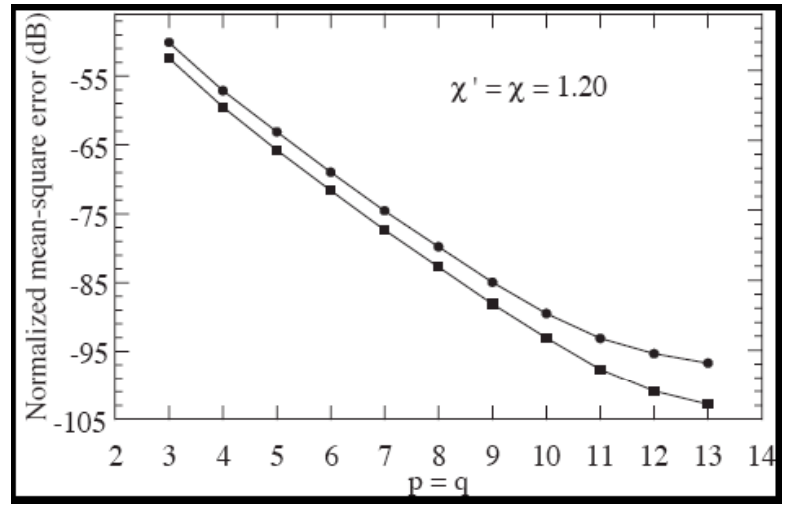

Fig. (4). Normalized mean-square error in the recovery of the uniform samples of. $V^{\prime}$ Dots: displacements in $[-\Delta \xi / 2, \Delta \xi / 2]$, $\left[-\Delta \varphi_{k} / 2, \Delta \varphi_{k} / 2\right]$. Squares: displacements in $[-\Delta \xi / 4, \Delta \xi / 4]$, $\left[-\Delta \varphi_{k} / 4, \Delta \varphi_{k} / 4\right]$.

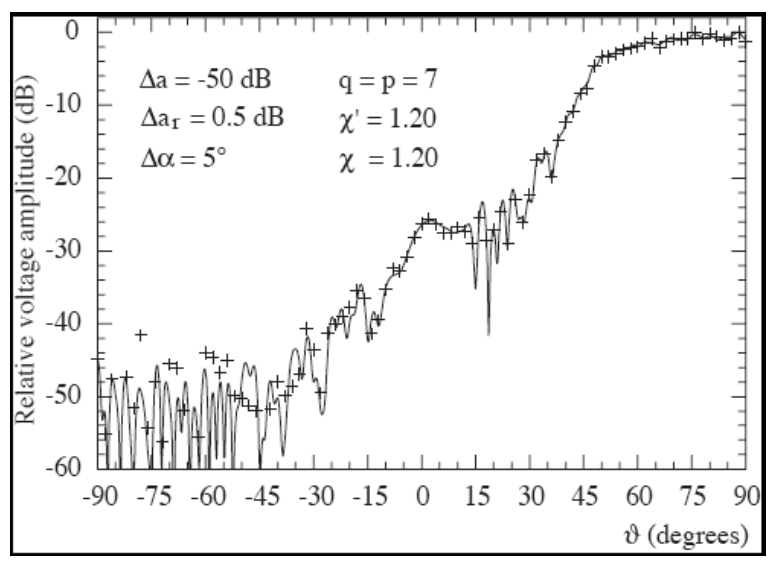

Fig. (5). Amplitude of the probe voltage $V^{\prime}$ on the meridian at $\varphi=90^{\circ}$. Solid line: exact. Crosses: reconstructed from error affected nonuniform samples via the SVD based algorithm.

these parallels are random variables uniformly distributed in $\left(-\Delta \varphi_{k} / 2, \Delta \varphi_{k} / 2\right)$. The amplitude and phase of the rotated probe voltage $V^{\prime}$ relevant to the meridian at $\varphi=90^{\circ}$ reconstructed via the SVD-based approach are shown in (Figs. 2 and 3). As can be seen, although the considered values of probe positioning errors are very pessimistic in an actual scanning, there is an excellent agreement between the reconstructed voltage (crosses) and the exact one (solid line). To assess the algorithm performances in a more quantitative way, the mean-square error in the reconstruction of the uniform samples has been evaluated. It is normalized to the voltage maximum value on the sphere and has been obtained by comparing the reconstructed and the exact uniform samples on the scanning sphere. Fig. (4) shows this error for $\chi^{\prime}=\chi=1.20$ and $p=q$ ranging from 3 to 13 . As can be seen, it decreases up to very low values on increasing the number of retained samples, thus assessing the effectiveness of the procedure. Even better results are to be expected when the distances between the position of each nonuniform sample and the associate uniform one are smaller. This is confirmed by the results shown in the same figure, which are relevant to the error in the reconstruction of the uniform samples when the displacements are random variables uniformly distributed in $(-\Delta \xi / 4, \Delta \xi / 4)$ and $\left(-\Delta \varphi_{k} / 4, \Delta \varphi_{k} / 4\right)$. The algorithm stability has been assessed (see Fig. 5) by adding random errors to the exact samples. These errors simulate a background noise (bounded to $\Delta a$ in amplitude and with arbitrary phase) and uncertainties on the data of $\pm \Delta a_{r}$ in amplitude and $\pm \Delta \alpha$ in phase. As expected (see Fig. 6), the stability can be improved by exploiting the redundancy for filtering the errors affecting the data. The same nonuniform NF data (used in Fig. 2) have been employed to reconstruct the voltage on the meridian at $\varphi=90^{\circ}$ by using the iterative approach (see Fig. 7). As can be seen, the reconstruction obtained by employing 5 iterations coincides with that relevant to the use of the SVD approach. Moreover, in order to give an insight on the convergence of the technique, the intermediate results when no iterations are used $(v=0)$ are shown in the same figure. The described SVD based procedure has been applied to efficiently recover the NF data needed to perform the NF-FF transformation. The reconstructed FF patterns in the principal planes $\mathrm{E}$ and $\mathrm{H}$ are compared with the exact ones in Figs. (8 and 9). As can be seen, the exact and recovered fields are practically indistinguishable, thus assessing the effectiveness of the technique. Identical results (not reported here for space saving) are obtained when the NF data required to carry out the transformation are retrieved from the same nonuniform NF data via the iterative approach.

In the second set of figures (from Fig. 10 to Fig. 15), the hypothesis of nonuniform sampling points lying on parallels is removed. Accordingly, the nonuniform samples have been generated in such a way that the distances in $\xi$ and $\varphi$ between the position of each nonuniform sample and the associated uniform one are random variables uniformly distributed in $(-\Delta \xi / 3, \Delta \xi / 3)$ and $\left(-\Delta \varphi_{n} / 3, \Delta \varphi_{n} / 3\right)$. The amplitude and phase of the rotated probe voltage relevant to the meridian at $\varphi=90^{\circ}$, reconstructed via the iterative technique, are compared in Figs. (10 and 11) with the exact ones. 


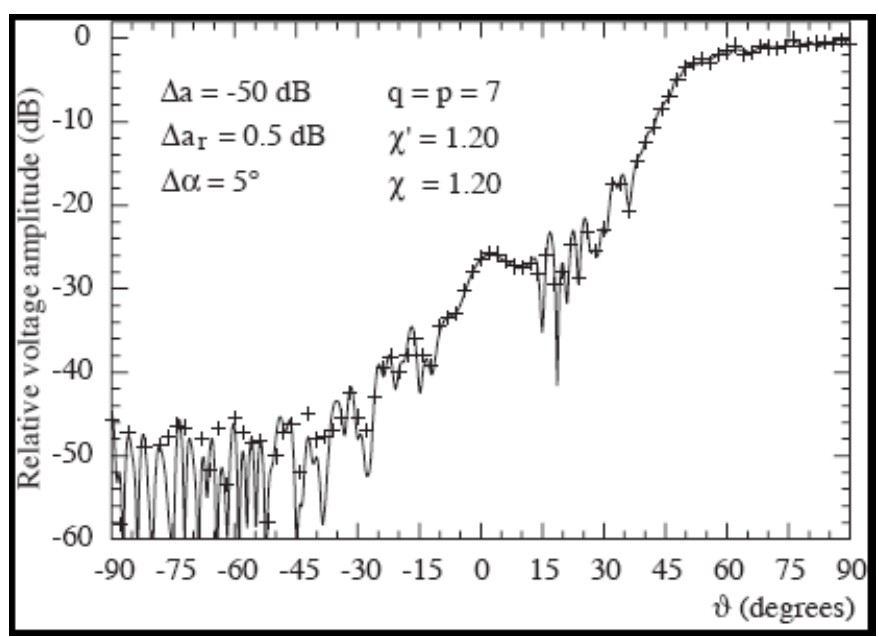

Fig. (6). Amplitude of the probe voltage $V^{\prime}$ on the meridian at $\varphi=90^{\circ}$. Solid line: exact. Crosses: reconstructed from error affected nonuniform samples (increased by $20 \%$ ) via the SVD based algorithm.

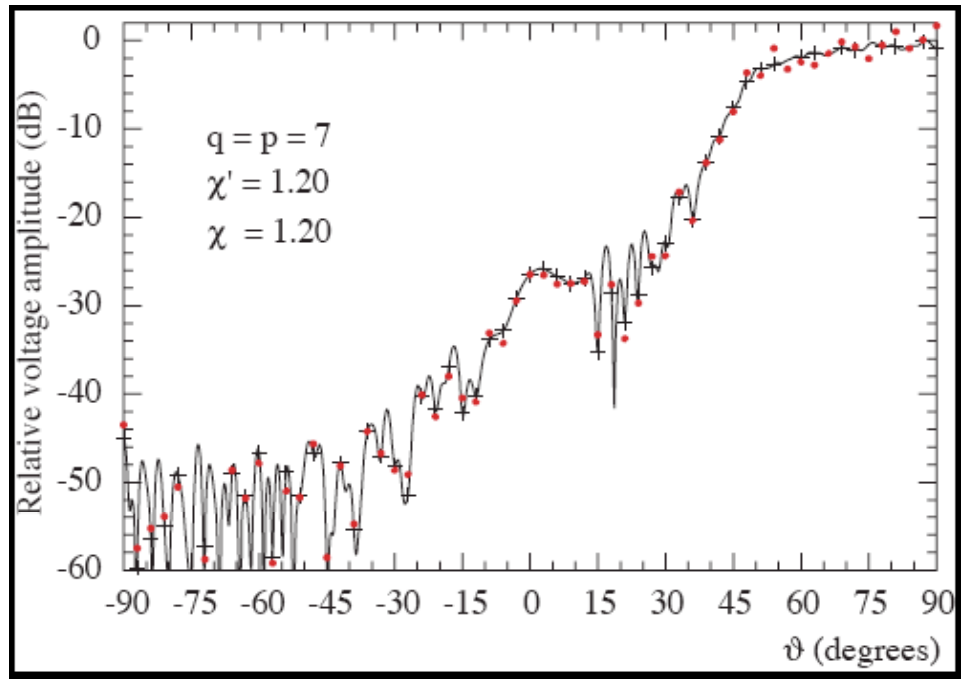

Fig. (7). Amplitude of the probe voltage $V^{\prime}$ on the meridian at $\varphi=90^{\circ}$. Exact: solid line. Reconstructed from nonuniform samples via the iterative algorithm: red dots for $v=0$ and black crosses for $v=5$.

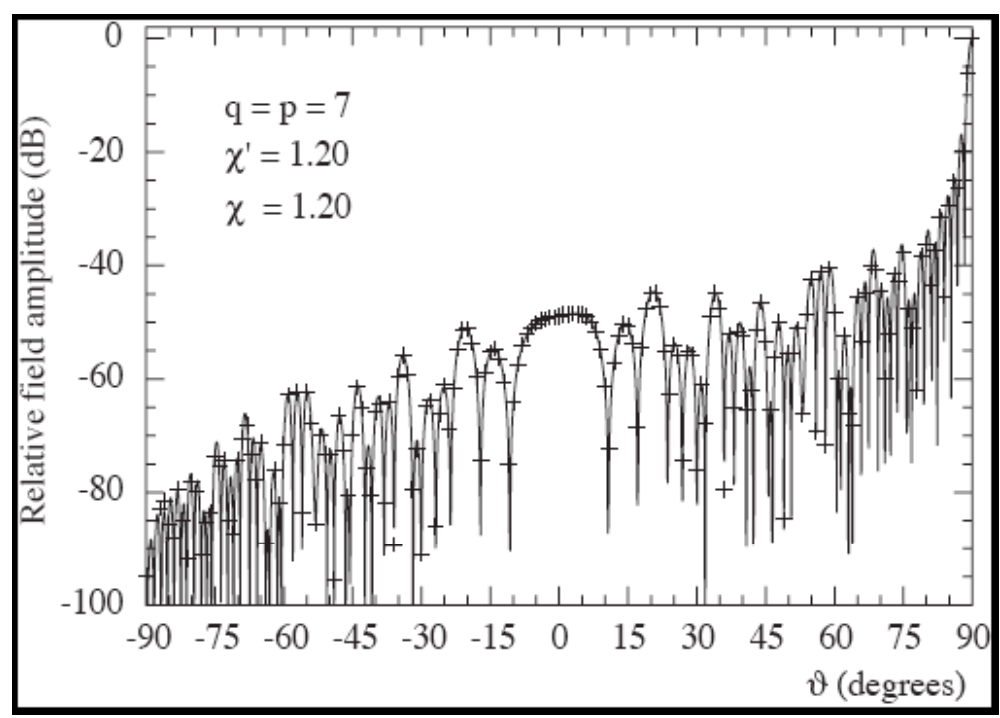

Fig. (8). Far-field pattern in the E-plane. Solid line: exact. Crosses: reconstructed from irregularly spaced NF samples via the SVD based algorithm. 


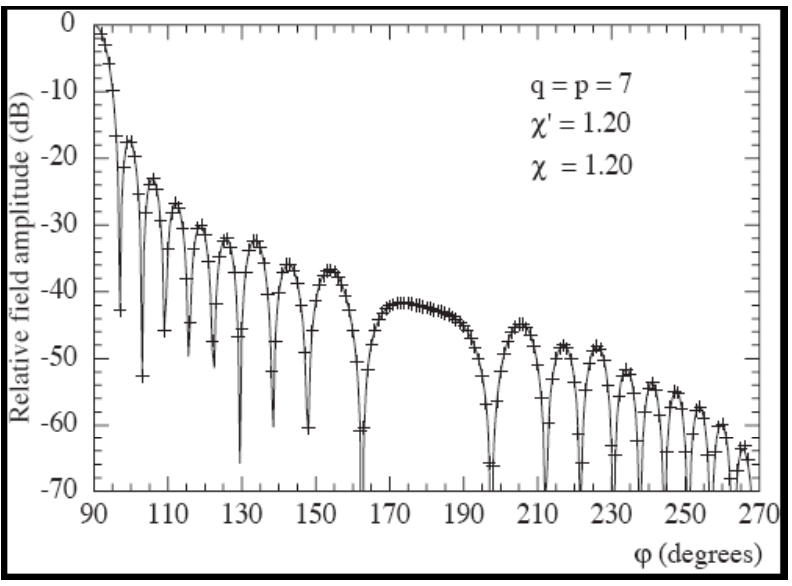

Fig. (9). Far-field pattern in the H-plane. Solid line: exact. Crosses: reconstructed from irregularly spaced NF samples via the SVD based algorithm.

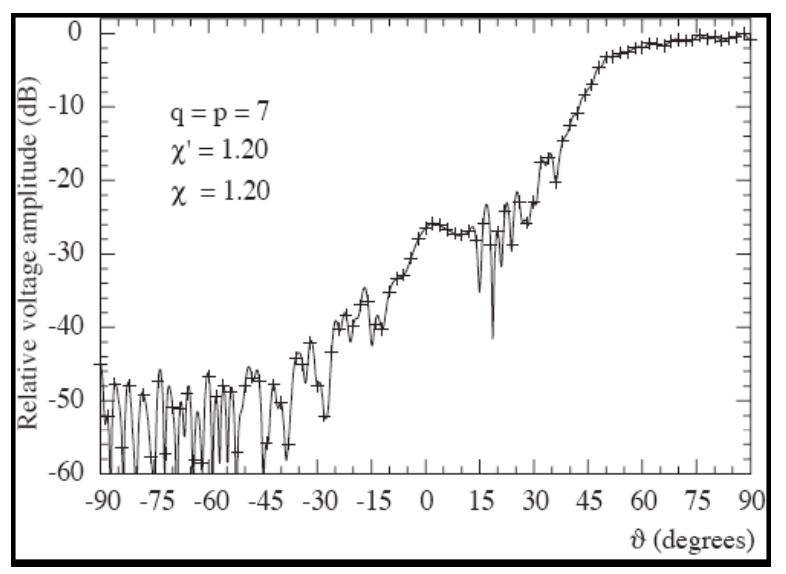

Fig. (10). Amplitude of the probe voltage $V^{\prime}$ on the meridian at $\varphi=90^{\circ}$. Solid line: exact. Crosses: reconstructed from nonuniform samples via the iterative algorithm $(v=5)$.

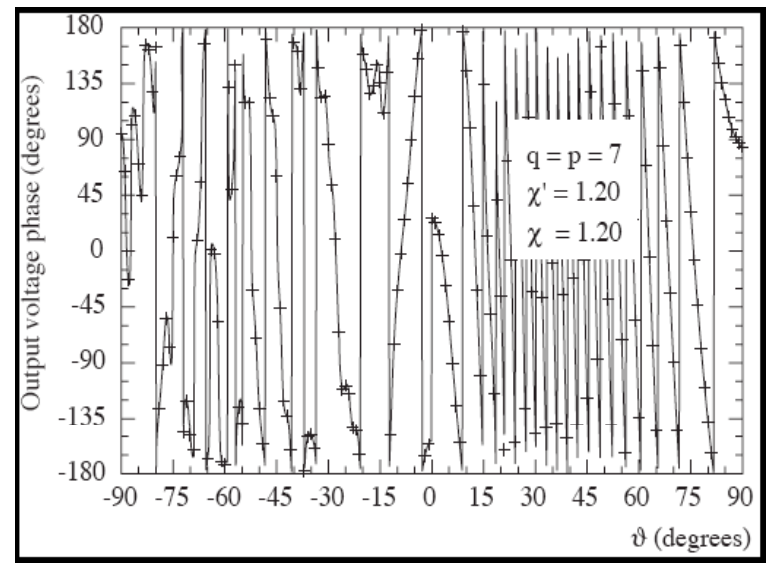

Fig. (11). Phase of the probe voltage $V^{\prime}$ on the meridian at $\varphi=90^{\circ}$. Solid line: exact. Crosses: reconstructed from nonuniform samples via the iterative algorithm $(v=5)$.

The evaluation of the mean-square error (normalized to the maximum value of $V^{\prime}$ on the sphere) in the reconstruction of the uniform samples assesses more quantitatively the effectiveness of the iterative algorithm (Fig. 12). As can be seen, on increasing the number of iterations, the error decreases

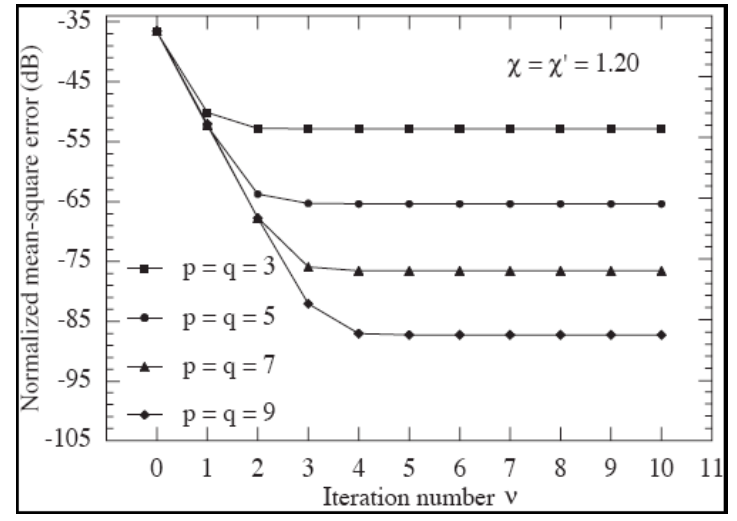

Fig. (12). Normalized mean-square error in the reconstruction of the uniform samples of $V^{\prime}$ when using the iterative algorithm as function of $v$.

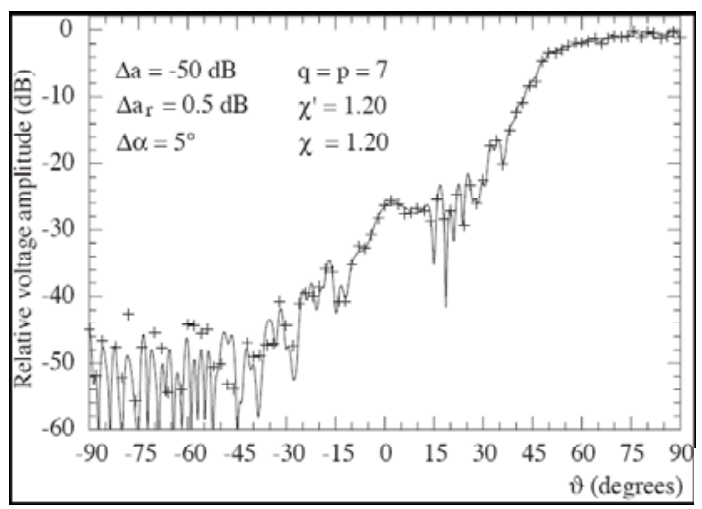

Fig. (13). Amplitude of the probe voltage $V^{\prime}$ on the meridian at $\varphi=90^{\circ}$. Solid line: exact. Crosses: reconstructed from error affected nonuniform samples via the iterative algorithm $(v=5)$.

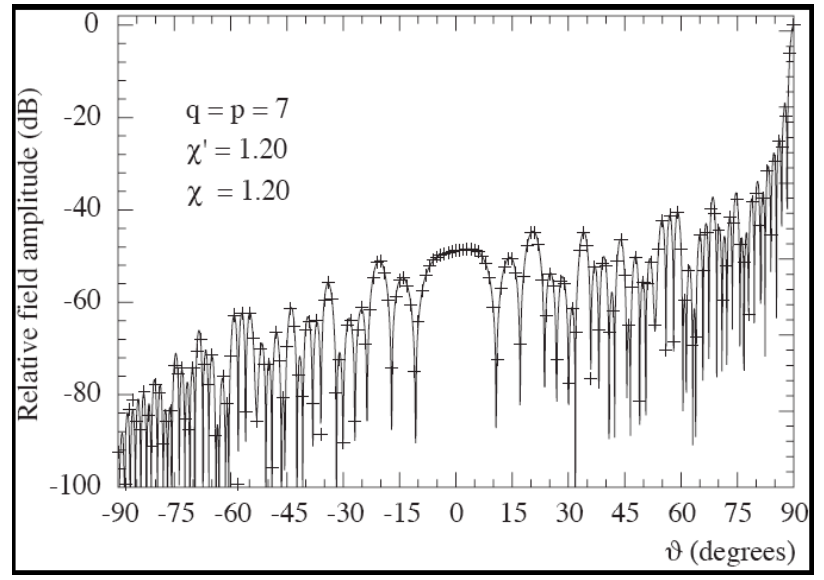

Fig. (14). Far-field pattern in the E-plane. Solid line: exact. Crosses: reconstructed from irregularly spaced NF samples via the iterative algorithm $(v=5)$.

quickly until a constant saturation value is reached. Such a value decreases on increasing the retained samples number. Also in this case, the robustness of the algorithm has been assessed. As can be seen in Fig. (13), the algorithm exhibits a good rejection to the data noise. At last, Figs. (14 and 15) show the antenna FF pattern in the principal planes $\mathrm{E}$ and $\mathrm{H}$ reconstructed from the irregularly distributed samples. As can be seen, the exact and recovered patterns are practically 


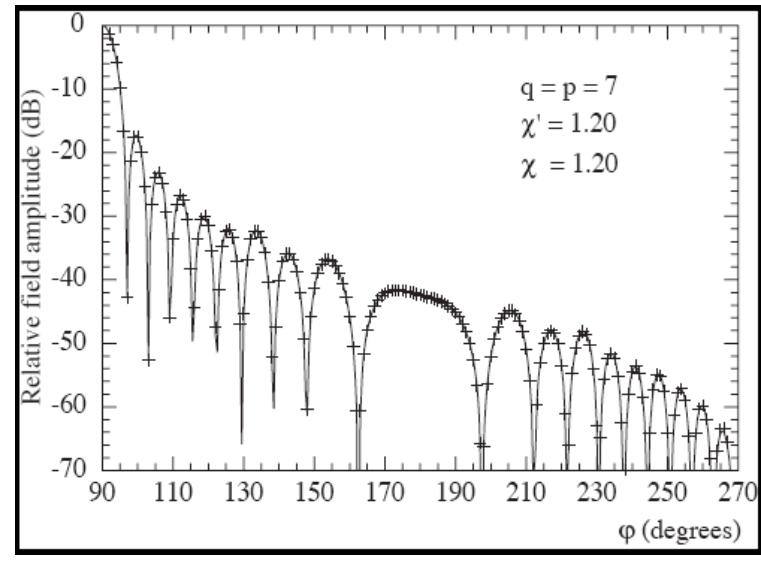

Fig. (15). Far-field pattern in the H-plane. Solid line: exact. Crosses: reconstructed from irregularly spaced NF samples via the iterative algorithm $(v=5)$.

indistinguishable, thus providing an overall assessment of the described iterative technique.

It can be interesting to compare the number of the employed nonuniform NF data (9 139) with those (130 562) needed by the classical NF-FF transformation with spherical scanning [1].

\section{CONCLUSIONS}

In this paper, two different techniques to compensate the probe positioning errors in a nonredundant NF-FF transformation with spherical scanning have been presented. The former makes use of the SVD method for recovering the uniformly distributed samples from the irregularly spaced ones and has been applied when the two-dimensional problem can be reduced to find the solution of two independent one-dimensional ones. The latter employs an iterative technique which requires a biunique correspondence, associating at each uniform sampling point the nearest nonuniform one. Although the considered positioning errors are very pessimistic in an actual scanning, excellent results have been achieved both in the NF and FF reconstructions.

\section{REFERENCES}

[1] J. Hald, J.E. Hansen, F. Jensen, and F.H. Larsen, Spherical nearfield antenna measurements. J.E. Hansen Ed., IEE Electromagnetic Waves Series, London: Peter Peregrinus, 1998.

[2] F. Jensen, "Electromagnetic near-field-far-field correlations," Ph.D. dissertation, Technical University of Denmark, Rep. LD15, 1970.

[3] P.F. Wacker, "Non-planar near-field measurements: Spherical scanning," NBSIR 75-809, Boulder, CO, 1975.

[4] F.H. Larsen, "Probe correction of spherical near-field measurements," Electron, Lett., vol. 13, pp. 393-395, July 1977.

[5] F.H. Larsen, "Probe-corrected spherical near-field antenna measurements," Ph.D. dissertation, Technical University of Denmark, Rep. LD36, 1980.

[6] A.D. Yaghjian, and R.C. Wittmann, "The receiving antenna as a linear differential operator: application to spherical near-field measurements," IEEE Trans. Antennas Propagat., vol. AP-33, pp. 1175-1185, November 1985.

[7] J.E. Hansen, and F. Jensen, "Spherical Near-Field Scanning at the Technical University of Denmark," IEEE Trans. Antennas Propagat., vol. AP-36, pp. 734-739, June 1988.

[8] O.M. Bucci, F. D’Agostino, C. Gennarelli, G. Riccio, and C. Savarese, "Data reduction in the NF-FF transformation technique with spherical scanning," J. Electromagn. Waves Appl., vol. 15, pp. 755-775, 2001.

[9] A. Arena, F. D'Agostino, C. Gennarelli, and G. Riccio, "Probe compensated NF-FF transformation with spherical scanning from a minimum number of data," Atti Fondazione G. Ronchi, vol. 59, pp 312-326, 2004.

[10] F. D'Agostino, F. Ferrara, C. Gennarelli, R. Guerriero, and M. Migliozzi, "Effective antenna modellings for a NF-FF transformation with spherical scanning using the minimum number of data," Int. J. Antennas Propagat., vol. 2011, ID 936781, 11 pages.

[11] O.M. Bucci, and G. Franceschetti, "On the spatial bandwidth of scattered fields," IEEE Trans. Antennas Propagat., vol. AP-35, pp. 1445-1455, December 1987.

[12] O.M. Bucci, C. Gennarelli, and C. Savarese, "Representation of electromagnetic fields over arbitrary surfaces by a finite and nonredundant number of samples," IEEE Trans. Antennas Propagat., vol. 46, pp. 351-359, March 1998.

[13] A. Dutt, and V. Rohklin, "Fast Fourier transforms for nonequispaced data," Proc. of SIAM J. Scie. Comput., vol. 14, pp. 13691393, November 1993.

[14] G. Beylkin, "On the fast Fourier transform of functions with singularities," Appl. Comput. Harmonic Anal., vol. 2, pp. 363-381, 1995.

[15] R.C. Wittmann, B.K. Alpert, and M.H. Francis, "Near-field antenna measurements using non-ideal measurement locations," IEEE Trans. Antennas Propagat., vol. 46, pp. 716-722, May 1998.

[16] R.C. Wittmann, B.K. Alpert, and M.H. Francis, "Near-Field, spherical scanning antenna measurements with nonideal probe locations," IEEE Trans. Antennas Propagat., vol. 52, pp. 2184-2186, August 2004

[17] O.M. Bucci, C. Gennarelli, and C. Savarese, "Interpolation of electromagnetic radiated fields over a plane from nonuniform samples," IEEE Trans. Antennas Propagat., vol. AP-41, pp. 15011508, November 1993.

[18] O.M. Bucci, C. Gennarelli, G. Riccio, and C. Savarese, "Electromagnetic fields interpolation from nonuniform samples over spherical and cylindrical surfaces," IEE Proc. Microw. Antennas Propagat., vol. 141, pp. 77-84, April 1994.

[19] F. Ferrara, C. Gennarelli, G. Riccio, and C. Savarese, "Far field reconstruction from nonuniform plane-polar data: a SVD based approach," Electromagnetics, vol. 23, pp. 417-429, July 2003.

[20] F. Ferrara, C. Gennarelli, G. Riccio, and C. Savarese, "NF-FF transformation with cylindrical scanning from nonuniformly distributed data," Microw. Optical Tech. Lett., vol. 39, pp. 4-8, October 2003.

[21] F. D'Agostino, F. Ferrara, C. Gennarelli, R. Guerriero, and M. Migliozzi, "On the compensation of probe positioning errors when using a nonredundant cylindrical NF-FF transformation," Prog. Electromagn. Res. B, vol. 20, pp. 321-335, 2010.

[22] G.H. Golub, and C.F. Van Loan, Matrix computations, Baltimore: J. Hopkins University Press, 1996.

[23] F. D'Agostino, F. Ferrara, C. Gennarelli, R. Guerriero, and M. Migliozzi, "Near-Field/Far-Field transformation with helicoidal scanning from irregularly spaced data," Int. J. Antennas Propagat., vol. 2010, ID 859396, 8 pages.

[24] O.M. Bucci, G. D’Elia, and M.D. Migliore, "Advanced field interpolation from plane-polar samples: experimental verification," IEEE Trans. Antennas Propagat., vol. 46, pp. 204-210, February 1998.

(C) D’ Agostino et al.; Licensee Bentham Open.

This is an open access article licensed under the terms of the Creative Commons Attribution Non-Commercial License (http://creativecommons.org/licenses/by-nc/3.0/) which permits unrestricted, non-commercial use, distribution and reproduction in any medium, provided the work is properly cited. 